\title{
Comparing Wealth Polarization Over Time and Across Countries in Africa
}

\author{
AbdelRahmen El Lahga* \\ Institut Supérieur de Gestion, Tunis, Tunisia. \\ Email: abdelrahmen.ellahga@gnet.tn \\ Preliminary and incomplete \\ (This version: April 2005)
}

\begin{abstract}
In this paper we compare wealth polarization within and across ten African countries. Using DHS data and applying the recently developed polarization measure by Duclos et al (2004) we find that most countries included in this study experienced a sharp decrease of polarization but at different rates. Compared to inequality, polarization behaves differently suggesting the different sensitivities of both notions to changes in different parts of the distribution of wealth. Our decomposition of polarization by ethnic, religious and wealth groups shows that: In general, the relative contribution of the poorest population to the overall polarization is greater than their relative size. In all countries the relative contribution of each ethnic or religious group to the overall polarization is very close to its relative size.
\end{abstract}

*The author gratefully acknowledges useful comments by Sami Bibi and Rim Chatti. 


\section{Introduction}

Conceptualized and measured by Esteban and Ray (1994), Wolfson (1994), and Duclos, Esteban and Ray (2004), polarization of an income distribution has recently received lots of attention from economic literature. ${ }^{1}$ Roughly speaking, income polarization means the extent to which a population is clustered around a small number of distant poles. The concept of polarization is fundamentally different from that of inequality. Indeed, a population displaying high inequality, with few persons appropriating most income, is not a polarized society, simply because most people are concentrated around a same pole in the income space. ${ }^{2}$ The conjecture that motivates studies of polarization is that the more polarized a society is, the more likely it seems that a conflict can break out. Consequently, finding an increase of income polarization can be a mean to detect and predict possibilities for social conflict and revolutionary tendencies.

Most empirical studies of income polarization have been applied to developed countries. However, according to the The PRIO/Uppsala Armed Conflict Dataset, ${ }^{3}$ among 275 internal conflicts recorded in the world, during the period going from 1993 to 2003, 120 occurred in sub-sahara Africa, making it advisable to question the trends of wealth polarization in this region of the world. The first goal of this paper is to fill in some blank spaces by measuring and comparing wealth polarization within and across 10 sub-saharan African countries, using a polarization measure proposed by Duclos, Esteban and Ray (2004) - "DER" hereafter.

Most of countries included in this study are characterized by ethnic and religious diversity which could lead to political instability and civil war(reference ??). We will emphasize, when it's relevant, the relative contribution of each ethnic group to the overall polarization of each country. Thus we offer an empirical framework of an unified treatment of wealth polarization and ethnic heterogeneity in a given country.

As we will see below DER's polarization measure is defined as the sum of antagonism-caused by the income difference-felt by each individual in the society. One could speculate that the relative antagonism felt by the poorest population may be more proportional to their relative size. The second goal of this paper, therefore, is to decompose overall polarization displayed by a given population by income percentile in order to better analyze the contri-

\footnotetext{
${ }^{1}$ See, also, Wang and Tsui (2000), Chakravarty and Majumder (2001), Zhang and Kanbur (2001), Rodriguez and Salas (2002) for others extensions and measurements of polarization.

${ }^{2}$ See aforementioned papers for several illustrative examples confirming this assertion.

${ }^{3}$ Data can be downloaded from http://www.prio.no/jpr/datasets.asp.
} 
bution of each income groups to the polarization. We propose in the same line of reasoning, a novel curve which we will call Cumulative Polarization Curve "CPC" that give a graphical illustration of antagonism felt by each percentile of income distribution. When addressing this issue we can improve our understanding of several aspects of social, economic and political changes on the continent.

A pre-requisite to our empirical analysis is a clear definition of what we mean by household wealth. Unlike the widely used procedure that proxies household wealth by current income or expenditure, we generate an index based on asset ownership (e.g, owning Tv, radio) and housing characteristics (e.g,type of toilet facilities, floor material) as an alternative proxy of household wealth. Our choice is motivated by several technical and conceptual problems that arise if we use income or expenditure as an indicator of household wealth.

At the technical level, while many of household income and expenditures surveys are available for African countries, using these surveys to make intertemporal comparisons of wealth polarization within and across countries is problematic. Indeed, as documented by Sahn and Stifel (2003) in more detail, the continual changes in surveys designs (e.g., the recall period, the number and choice of item codes listed...), the absence of valid price indices and rental market, self-employment and seasonal variability in earnings can have a non negligible effect on the measurement of household expenditures or income.

At the conceptual level, and at least since Sen's (1985) paper, it is common to argue that income covers one aspect of living standard. Others aspects should be included in the evaluation of well-being, like living conditions and access to basic facilities. Even if you have enough income, but you live in an unsanitary house, you will obtain a little benefit from your expenditures. Thus, the index based on asset ownership can be considered as a logical application, even partial, of Sen's viewpoint.

The approach considering asset based index as a proxy of household wealth has been previously used by Filmer and Pritchett (2001) and Sahn and Stifel (2000). They create an asset index based on a set of dichotomous variables available in the Demographic and Health Surveys (DHS), and then use that index to study respectively educational enrollments in India and poverty in Africa. Beyond the merits of using such index, Sahn and Stifel (2003) show, in a comparative study, that the asset index performs as well, if not better than reported expenditures in predicting children's nutritional status.

The following Section presents DER (2004) measure of polarization as well as the decomposition of polarization measure by sub-population and presents our "CPC". Section 3 deal in more detail with the methods employed to 
construct the welfare index used in our analysis and the data we use. Section 4 reports trends in polarization within and across countries. We conclude by summarizing the major findings.

\section{Income Polarization According to DER (2004)}

\section{$2.1 \quad$ A polarization measure}

Let us consider an income distribution defined by a density $f$ over $\mathbb{R}_{+}$. DER assume that each individual feels two things: Identification with similar people, and an alienation from dissimilar people. For someone with income $x$ the sense of identification depends on the density $f(x)$ at $x$. For two people with incomes $x$ and $y$, the sense of alienation is monotonic in distance $|x-y|$. The interaction between both feelings gives rise to the effective antagonism of $x$ towards $y$ (under $f$ ). Such antagonism can be written as some function

$$
T(i, a)
$$

where $i=f(x), a=|x-y|$, and $T$ is some arbitrary continuous and non decreasing function with $T(i, 0)=T(0, a)=0$. Polarization is defined as the addition of all effective antagonisms in the distribution:

$$
P(f)=\iint T(i, a) f(x) f(y) d y d x
$$

Last equation gives a general class of polarization measures that fits into what DER call the "identification-alienation" framework. The approach in DER places a set of four axioms on this framework so as to narrow down a functional form of $T$ and gives the following useable measure of polarization:

$$
P_{\alpha}(f) \equiv \iint f(x)^{1+\alpha} f(y)|y-x| d y d x
$$

where $\alpha \in[.25,1]$ indicates the degree of polarization aversion displayed by the measure.

For the empirical estimation of (2), DER note, first, that for every distribution function $F$ with associated density $f$ and mean $\boldsymbol{\mu}$, equation (2) can be rewritten as:

$$
P_{\alpha}(F)=\int_{y} f(y)^{\alpha} a(y) d F(y),
$$

with $a(y) \equiv \boldsymbol{\mu}+y(2 F(y)-1)-2 \int_{-\infty}^{y} x d F(x)$. A natural estimator of $P_{\alpha}(F)$ based on a random sample of $n$ i.i.d observations of income $y_{i}, i=1 \ldots n$ and 
ordered such that $y_{1} \leq y_{2} \leq \ldots \leq y_{n}$, is

$$
P_{\alpha}(\widehat{F})=n^{-1} \sum_{i=1}^{n} \widehat{f}\left(y_{i}\right)^{\alpha} \widehat{a}\left(y_{i}\right)
$$

where $\widehat{a}\left(y_{i}\right)$ is given as

$$
\widehat{a}\left(y_{i}\right)=\widehat{\boldsymbol{\mu}}+y_{i}\left(n^{-1}(2 i-1)-1\right)-n^{-1}\left(2 \sum_{j=1}^{i-1} y_{j}+y_{i}\right),
$$

$\widehat{\boldsymbol{\mu}}$ is the sample mean, and where $\widehat{f}\left(y_{i}\right)^{\alpha}$ is the nonparametric kernel estimator of $f\left(y_{i}\right)^{\alpha}$.

DER show that $n^{5}\left(P_{\alpha}(\widehat{F})-P_{\alpha}(F)\right)$ has an asymptotic limiting normal distribution $N\left(0, V_{\alpha}\right)$, with

$V_{\alpha}=\operatorname{var}\left((1+\alpha) f(y)^{\alpha} a(y)+y \int f(x)^{\alpha} d F(x)+2 \int_{y}^{\infty}(x-y) f(x)^{\alpha} d F(x)\right)$.

The last result will be used to make statistical comparisons of wealth polarization within and between countries.

\subsection{Decomposing the polarization measure}

\subsubsection{Decomposing polarization by sub-population}

Given equation (4) polarization index $P_{\alpha}(\widehat{F})$, which we note $P$ for expositional simplicity, can be rewritten as :

$$
P=n^{-1} \sum_{i=1}^{n} c_{i}
$$

where $c_{i}=\widehat{f}\left(y_{i}\right)^{\alpha} \widehat{a}\left(y_{i}\right)$. We can interpret $c_{i}$ as the contribution of individuals with income $y_{i}$ to the overall polarization or equivalently antagonism felt by an individual $i$. Consider now any characteristic, e.g. ethnicity, region or income intervals, yielding a partition of the whole population in $K$ groups each with size $n_{k}$, with $\sum n_{k}=n$ and $k=1 \ldots K$. Equation (6) can be then rewritten as:

$$
P=n^{-1} \sum_{j=1}^{k} \sum_{i=1}^{n_{k}} c_{i}^{k}
$$


with $c_{i}^{k}$ denote antagonism felt by an individual $i$ in group $k, \sum_{i=1}^{n_{k}} c_{i}^{k}$ is the contribution of group $k$ to the overall polarization $P$. The relative contribution of group $k$ is, consequently, given by:

$$
C(k)=\frac{\sum_{i=1}^{n_{k}} c_{i}^{k}}{\sum_{i=1}^{n} c_{i}} .
$$

$C(k)$ can be compared to the population share of the group $k$. In the empirical illustration, of this paper we will compare the relative contribution of each ethnic group to its relative size in the whole population. The idea here is when a population displays a "moderate" polarization the contribution of each group should be close to its relative size.

Another interesting case occurs when we define groups in terms of income or wealth. Recall that the discrete income values $y_{i}$ are ordered such that $y_{1} \leq y_{2} \leq \ldots \leq y_{n}$. Let $p \in[0,1]$ be the proportion of individuals in the population who enjoy a level of income that is less than or equal to $y$, and let $\operatorname{int}(z)$ be the largest integer less than or equal to $z$. The order of the observation corresponding to the percentile $p$ is defined by $\kappa(n, p)=\operatorname{int}[(n-1) p+1]$.

The relative contribution of the poorest $100 p \%$ of the population to the overall polarization is given by

$$
C(p)=\frac{\sum_{i=1}^{\kappa(n, p)} c_{i}}{\sum_{i=1}^{n} c_{i}} .
$$

Letting $p$ vary from zero to one $C(p)$ traces out what we will call "Cumulative Polarization Curve" (CPC). As we can see in Figure 1, where the CPC $M P L(p)$ and $C P C_{a}$ have been drawn for two hypothetical distribution $\mathrm{A}$ and $\mathrm{B}$, diagonal line $M P L(p)=p$ represents an income distribution with a moderate polarization i.e., the relative contribution of each proportion of the whole population is equal to its relative size $p$. The curve $C P C_{a}$ represents a distribution where the $100 p \%$ poorest members of the population contribute more proportionally than their relative size to the overall polarization.

Now it could be interesting to plot in the same figure the CPC and Lorenz curve. Recall that $L(p)$ indicates the cumulative percentage of total income held by a cumulative proportion $p$ of the population, when individuals are ordered in increasing values of their income. $L(p)$ can be interpreted as an alienation sentiment felt by the proportion $p$ of the population, and $C(p)$ as noted above is the antagonism felt by this same proportion $p$. We can compare $L(p)$ and $C(p)$ to the diagonal line which denote simultaneously perfect 


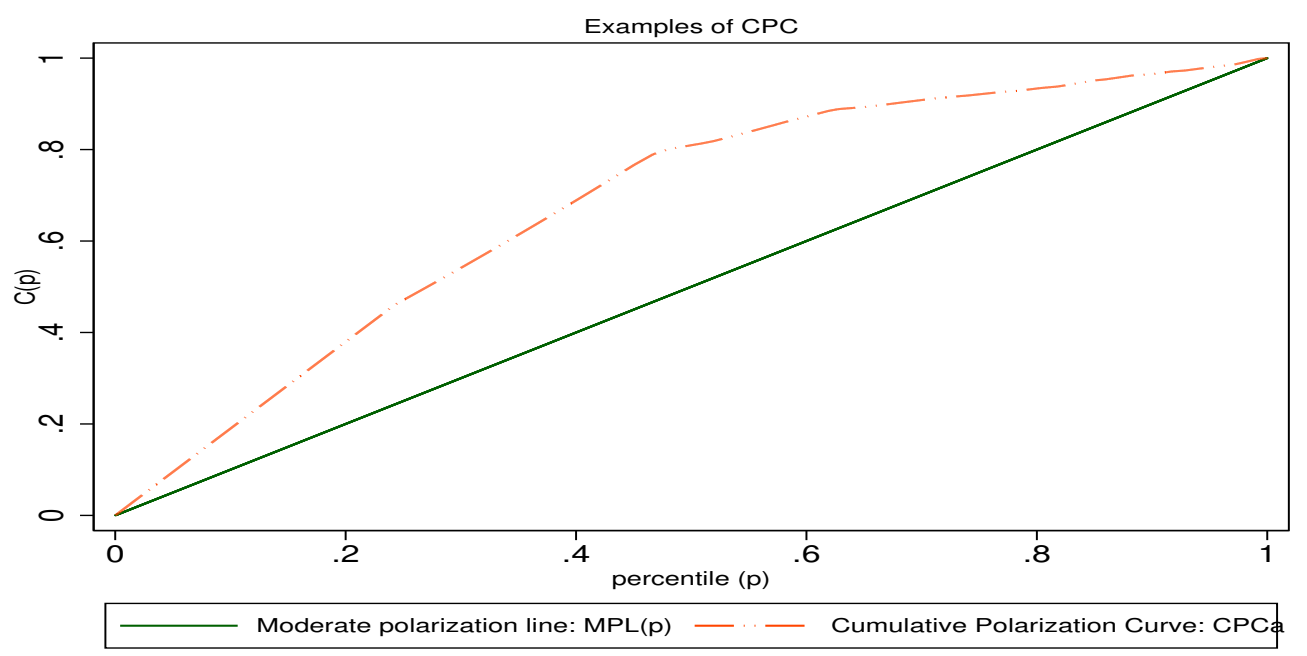

Figure 1: Examples of CPC

equality and moderate polarization displayed by a given distribution.

\subsubsection{Decomposing inter-temporal evolution of polarization index}

The central idea of DER's paper is that polarization is driven by the interplay of two forces: identification with one's own group and alienation vis-a-vis others. When analyzing an inter-temporal variation of polarization displayed by a given population, how we explain such changes in terms of identification and alienation sentiments? The answer is straightforward given the decomposition proposed by DER of polarization. Indeed DER show that, for a fixed value of $\alpha$, polarization $P$ is the product of average alienation, average identification, and (one plus) the mean normalized covariance between these two variable. Formally, we have

$$
P=\bar{\iota} \bar{a}(1+\rho),
$$

where $\bar{\iota}=\int f(y)^{1+\alpha} d y$ average identification, $\bar{a}=\int a(y) d F(y)$ is the average alienation which is twice the Gini coefficient, and $\rho=\frac{\operatorname{cov}(\iota, a)}{\bar{\iota} \bar{a}}$. Denote $1+\rho=$ $v$, and taking logarithm of (10) and differentiating it, we obtain

$$
\dot{P}=\dot{\bar{\iota}}+\dot{\bar{a}}+\dot{v}
$$

Equation (11) shows that relative change in polarization over time can be decomposed as the sum of three components: (i) the relative change of (av- 
erage) identification; the relative change of (average) alienation; and the relative change of their joint co-movement. Obviously, equation (11) is only valid for an infinitesimal change of $P$. In the empirical application we can obtain only an approximation of (11) given that we will consider a discrete change of $P$. Such rewriting of DER's decomposition may be useful in some cases to analyze changes in polarization index over time.

\section{Methodology and Data}

\subsection{Construction of an Asset Index}

Let us consider, $a_{i 1} \ldots a_{i K}, K$ variables describing ownership by household $(i)$ of asset $(k)$. To proxy wealth, we assume that the asset ownership of households closely reflects their living standard. Following Filmer and Pritchett (2001), and Sahn and Stifel (2000, 2003), the idea is to aggregate the data into a single composite asset index that contains most of the common information of the asset ownership. In others words, we want a linear asset index $A_{i}$, that proxies for household wealth, of the form

$$
A_{i}=\widehat{\gamma}_{1} \frac{a_{i 1}-\bar{a}_{1}}{\sigma_{1}}+\ldots+\widehat{\gamma}_{K} \frac{a_{i K}-\bar{a}_{K}}{\sigma_{K}},
$$

where $\widehat{\gamma}_{k}$ is the "scoring factor" or the weight assigned to asset $k$, which we must estimate, $\bar{a}_{k}$ is the mean value of $a_{i K}$ over all households, and $\sigma_{K}$ its standard deviation. Various options could be used to estimate or to impose weighting values of assets. ${ }^{4}$ In this paper we use the statistical procedure of Principal Component Analysis (PCA) to determine the weights $\widehat{\gamma}_{k}$. The first principal component will extract the largest amount of information contained in all these assets that accounts for the largest share of their variation. Our composite asset index is a good proxies of household's wealth if (and only if) the living standard is indeed the main determinant of asset variability among households.

Since we want to make inter-temporal comparisons of polarization and inequality for each country from surveys of different years, it is necessary to construct a wealth indexes that are comparable over time. To perform this task, the data sets for each country are pooled and the PCA scoring coefficients (asset weight) are estimated for the pooled sample. They are then applied to the separate samples to estimate the wealth indexes for each

\footnotetext{
${ }^{4}$ See for instance Filmer and Pritchett (2001), Sahn and Stifel (2000), Asselin (2002) and Kolenikov and Angeles (2004)
} 
of the households. For across countries comparisons, the latest surveys for each of the countries are pooled to estimate asset weights.

Some of the so created wealth indexes have negative values. This creates problems for our polarization and inequality analysis since theses indices are defined over positive real number. Any transformation used to define the distribution of the wealth index over supports in $\mathbb{R}_{+}$has an effect on the resulting inequality and polarization measures, as they preserve neither the mean nor the variance. To resolve this problem we follow previous work (e.g. Asselin 2002; Sahn and Stifel, 2003) that add a value equal to the greatest negative value to each of the asset indices, so that the lowest observed values become zero. This transformation implies that the values of the inequality and polarization indices do not have any meaning on their own, but only obtain meaning in the context of this research-i.e., for inter-temporal comparisons of inequality and polarization.

The assets included in the wealth index can be grouped into two categories: households durables and housing characteristics. The households durables consist of indicators of ownership of radios, TVs, refrigerators, motorized transportation (motorcycle and/or cars). The housing characteristics include indicators variables for source of drinking water (piped in house, well, and surface water relative to other sources), toilet facilities (flush toilet and no facilities relative to others facilities), an indicator for low quality of floors materials (earth floor) and one dummy variable if the household has access to electricity.

\subsection{Data}

For our purposes we use data drawn from the DHS conducted in the following 10 sub-saharan African countries, during the period 1988-2003: Burkina Faso (92-98), Ghana (93-98-2003), Côte d'Ivoire (94-99), Kenya (88-98), Mali (95-2001), Namibia (92-2000), Senegal (92-97), Tanzania (92-96), Uganda (95-2000) and Zimbabwe (94-99) ${ }^{5}$. The DHS, coordinated by Macro International, is an ongoing project that has been conducting household surveys in 65 developing countries worldwide since 1984. In 2004 around 150 surveys have been undertaken. The main purpose of the DHS surveys is to provide countries with the data needed to monitor and evaluate population, health and nutrition programs on a regular basis. For a given country, a typical self-weighted national sample of 5000 to 6000 households is selected and then

\footnotetext{
${ }^{5}$ Macro International, Inc implements the DHS programme with funding from USAID. All of these data and detailed information on surveys design can be downloaded from http://www.measuredhs.com
} 
interviewed using a household questionnaire to collect housing characteristics. Women between the ages 15 and 49 are interviewed using a women's questionnaire to collect information mainly on background characteristics, children and women's health, household assets and other issues, such as education level. ${ }^{6}$

A huge advantage of the DHS data files is that the questionnaires and most codes are unified across countries and surveys. Indicators for assets ownership have been generated on the basis of variables v119-v125 from DHS surveys. Housing characteristics have been generated on the basis of variables $v 113-v 116$ and $v 12 \%$.

\section{Results}

We report in table 1 weights from the principal components analysis (PCA) for each asset used in the construction of the wealth index. The PCA analysis assign, as expected, a positive weights for assets indicating greater wealth ( e.g., access to electricity) and a negative weights for variables indicating a lower wealth (e.g., no toilet facilities). Asset's weights are comparable across countries excepting Burkina Faso and Mali with a relatively low positive weights for the possession of flush toilet. Because all the asset variables take only the values 0 and 1 , the weights have an easy interpretation: a move from 0 to 1 changes the index by $\frac{\widehat{\gamma}_{i}}{\sigma_{i}}$ (see eq $(12)$ ).

Using the wealth index generated on the basis of the above step we estimate a polarization index $P_{\alpha}$, for $\alpha=1$, and Gini coefficient in the ten countries for each survey year. We set $\alpha=1$, the upper bound on $\alpha$, to give a large weight to polarization and to distinct it from Gini. To ease of comparisons, all indices are divided by 2 , so that $P_{\alpha=0}$ coincides with Gini coefficient.

\section{Changes in polarization and inequality over time}

Table 2 Shows estimation results of the two indices, along with their asymptotic standard deviation. Two features are apparent from table 2. First, all indices are estimated with high precision, with only the third decimal of the estimators being subject to sampling variability. Second, six (the first six countries listed in table 2) out of the ten countries experienced a statistically significant sharp reductions in both polarization and inequality, but at substantially different rates: polarization declines more faster than inequality,

\footnotetext{
${ }^{6}$ In some cases the sample size varies considerably, and some areas are over/under sampled. Household sampling weights are used to account for the over/under sampling.
} 
suggesting the different sensitivities of these two indices to changes in different parts of the distribution of wealth index. The case of Mali with 40.15\% reduction in polarization seems unrealistic and need a specific investigation in futures versions of this paper. For the others four countries polarization behaves differently from inequality. Burkina Faso witnessed between 1992 and 1998 a clear increases in inequality but polarization remains constant. In the case of Senegal and Tanzania, where inequality remained constant respectively during 1992-1997 and 1992-1996, polarization increased by $9.84 \%$ in Senegal and decreased by $15.99 \%$ in Tanzania. Ghana, the only country with 3 surveys in this study, experienced a sharp decrease in both inequality and polarization between 1993 and 2003, but during 1993-1998 the two indices behaved differently with clear increase in inequality by $5.86 \%$ and a decrease in polarization by $7.24 \%$.

To better understand inter-temporal changes in polarization we report in Table 3 the results of the decomposition of polarization index as the product of average alienation $\bar{a}$, average identification $\bar{\iota}$ and (one plus) the normalized covariance between the two $v$, as well as an approximation of equation (11) which decompose inter-temporal change of polarization. The interpretation of inter-temporal changes of polarization over time is straightforward for some countries. The sharp reduction of polarization in Ghana between 1998-2003 by $12.3 \%$ can be explained essentially by the net decline in average alienation while $\bar{\iota}$ and $v$ remained constant. The bulk of variation of polarization in Senegal $(+9.84 \%)$ stems from significant increase in average identification, with a non significant change of alienation and a small change of $v(-3.27 \%)$. In the case of Tanzania, where polarization decreased by $16.67 \%$, it was driven by net decrease of identification $(-6.28 \%)$ with non significant change of alienation and large change of $v(-11.05 \%)$. Burkina Faso the sole country where polarization remained constant, identification and alienation increased respectively by $7.68 \%$ and $9.54 \%$, but when taken jointly these two changes counterbalance each other to lead to a constant polarization.

In the others countries, where polarization decreased, identification and alienation moved in the same direction as polarization. In theses cases effects of identification and alienation reinforce each other and lead to a sharp reduction of polarization. In summary, in most countries included in this study polarization decreased substantially. Our empirical results show that in several cases polarization behaves differently from inequality and even when both measures move in the same direction their relative changes are substantially different.

Polarization by ethnic and religious groups

To illustrate decomposition of polarization by sub-population proposed in 
(8), we reports (when data are available) in table 4 the relative contribution of the two major ethnic and religious groups to the overall polarization compared to their relative size in the whole population ${ }^{7}$. Results show that the contributions of ethnic and religious groups are sensibly the same as their respective relative sizes. Consequently, in any of countries considered here, we can not conclude the existence of ethnic or religious group who feels an antagonism more proportional to it's size.

\section{Polarization by income percentiles}

We turn now to the presentation of our Cumulative Polarization Curve (CPC) for each country and for each survey year (figures 2-11). As can be seen in theses figures and for the first year survey of each country CPC is always above the Moderate Polarization Line MPL indicating that the antagonism felt by the poorest parts of the population is more proportional than their sizes. An interesting finding is that in general when we observe a decrease in polarization index between two years $t_{1}$ and $t_{2}$ the CPC of $t_{1}$ is always below that of $t_{2}$; and vice-versa when we observe an increase in polarization as in the case of Senegal. But in the case of Tanzania CPC of 1998 crosses twice that of 1992 indicating an increase to the contribution of the $40 \%$ poorest individuals to the overall polarization and a net reduction of the contribution of those between $40^{t h}$ and $67^{t h}$ percentile. In the cases of Côte d'Ivoire and Namibia their second year survey CPC cross the MPL at respectively the $47^{\text {th }}$ and $65^{\text {th }}$ percentile indicating the very low contribution of the population just above these percentile to the overall polarization. In summary CPC gives a net picture of the contribution of each income group to the overall polarization.

\section{Cross-country comparisons}

Table 5 presents the results of polarization and Gini indices for the ten countries based on the pooled wealth index distributions ${ }^{8}$, as well as the ranking of countries according to each index. Results show that the country with the lowest polarization is Côte d'Ivoire, followed by Mali, Ghana and Senegal. Polarization is highest in Tanzania, Namibia and Kenya. Nevertheless, Polarization induces very different ranking in comparison with inequality, except the case of Côte d'Ivoire which remains the more equal country in terms of wealth index. Tanzania moves from its the more polarized country to the sixth unequal country, the same remark for Namibia which moves from the

\footnotetext{
${ }^{7}$ Note that relative size of each group is calculated on the basis of sample used in this study and do not necessarily coincide with the real composition of the population

${ }^{8}$ We use the latest survey available for each countries to construct the wealth index. While the years of the surveys vary, they all fall within a span of five years, 1996-2001.
} 
rank 2 in terms polarization to rank 7 in terms of inequality. Theses findings confirm empirically the differences between polarization and inequality.

\section{Conclusion}

By using DHS data we have examined the degree of wealth polarization within and across ten Sub-Saharan African countries. Our empirical results show the sharp decrease of polarization in most countries included in this study. Compared to inequality, polarization behaves differently and confirm the conceptual difference between both notions. We have proposed a decomposition of DER polarization measure by sub-population and income percentiles. Our empirical illustrations show that in general antagonism felt by the poorest population is more proportional than their relative size.

\section{References}

[1] Collier, Paul and Anke Hoeffler, (2002) "Greed and Grievance in Civil War", manuscript, World Bank

[2] Chakravarty, S.R. and A. Majumder (2001), "Inequality, Polarization and Welfare: Theory and Applications," Australian Economic Papers 40, 1-13.

[3] D'Ambrosio, C. and E. Wolff (2001), "Is Wealth Becoming More Polarized in the United States?" Working Paper 330, Levy Economics Institute, Bard College.

[4] Deutsch, M. (1971) "Conflict and its Resolution" in Conflict Resolution: Contributions of the Behavior Sciences ed. by C. G. Smith. Notre Dame: University of Notre Dame Press.

[5] Dollar, David, and Aart Kraay, 2002, "Growth is Good for the Poor," Journal of Economic Growth, 7, 195-225.

[6] Duclos, J.Y., J.Esteban, and D. Ray (2004): "Polarization: Concepts,Measurement, Estimation," Econometrica 72, 1737-1772.

[7] Esteban, J. and D. Ray (1994) "On the Measurement of Polarization," Econometrica 62, 819-852.

[8] Esteban, J. and D. Ray (1999) "Conflict and Distribution," Journal of Economic Theory 87, 379-415. 
[9] Esteban, J., C. Gradn, and D. Ray (1999): "Extensions of a Measure of Polarization, with an Application to the Income Distribution of Five OECD Countries," Mimeo, Instituto de Analisis Economico.

[10] Gradn, C. (2000), "Polarization by Sub-Populations in Spain, 1973-91," Review of Income and Wealth 46, 457-474.

[11] Hegre, Håvard; Ranveig Gissinger and Nils Petter Gleditsch, (2003). "Globalization and Internal Conflict", in Gerald Schneider; Katherine Barbieri and Nils Petter Gleditsch, eds, Globalization and Armed Conflict. Oxford: Rowman and Littlefield.

[12] Milanovic, B. (2000), "A New Polarization Measure and Its Applications," mimeo., Development Research Group, World Bank.

[13] Pagan, A.R., and A. Ulluh (1999): Nonparametric Econometrics. Cambridge,MA: Cambridge University Press.

[14] Rodriguez, J.G. and R. Salas (2002), "Extended Bi-Polarization and Inequality Measures," mimeo, Universidad Complutense de Madrid.

[15] Sen, A. (1973): On Economic Inequality (Second Ed.). Oxford: Oxford University Press and Clarendon Press.

[16] Wang, Y.Q. and Tsui, K.Y. (2000), "Polarization Orderings and New Classes of Polarization Indices," Journal of Public Economic Theory 2, 349-363.

[17] Wolfson, M.C. (1994) "When Inequalities diverge," American Economic Review 84, Papers and Proceedings, 353-358.

[18] Wolfson, M.C. (1997) "Divergent Inequalities: Theory and Empirical Results," Review of Income and Wealth 43, 401-421.

[19] Zhang, X. and R. Kanbur (2001), "What Difference Do Polarisation Measures Make? An Application to China," Journal of Development Studies 37, 85-98. 


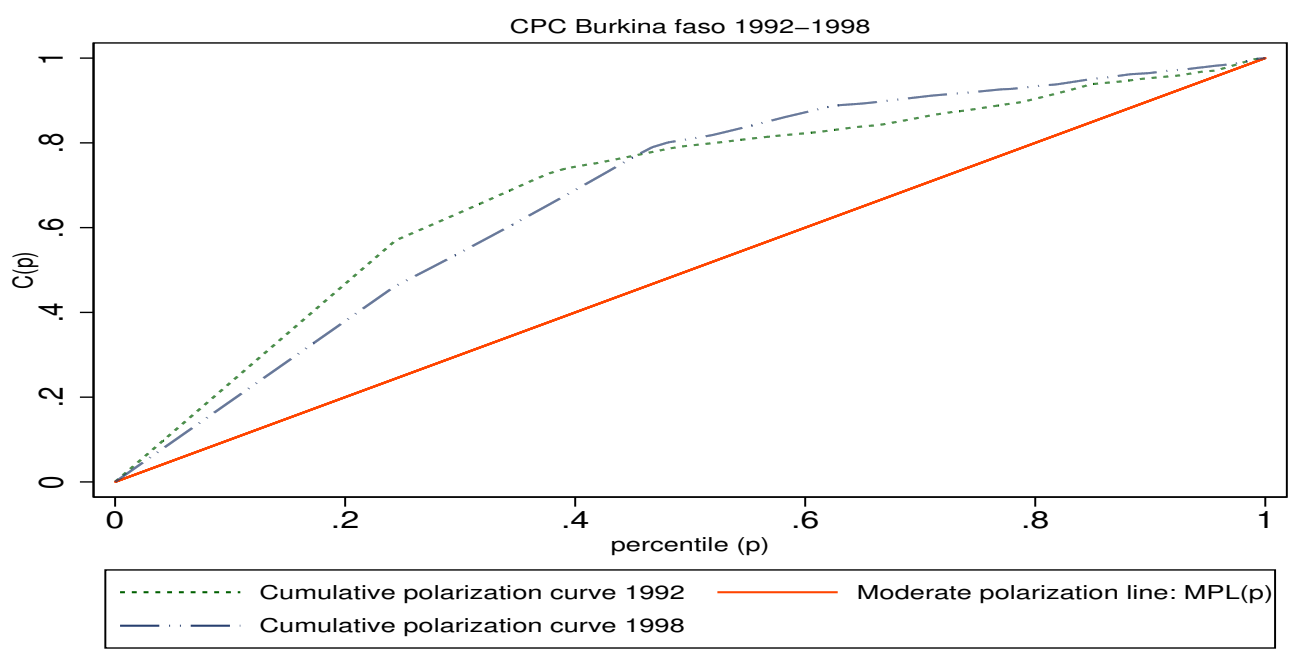

Figure 2: Burkina Faso

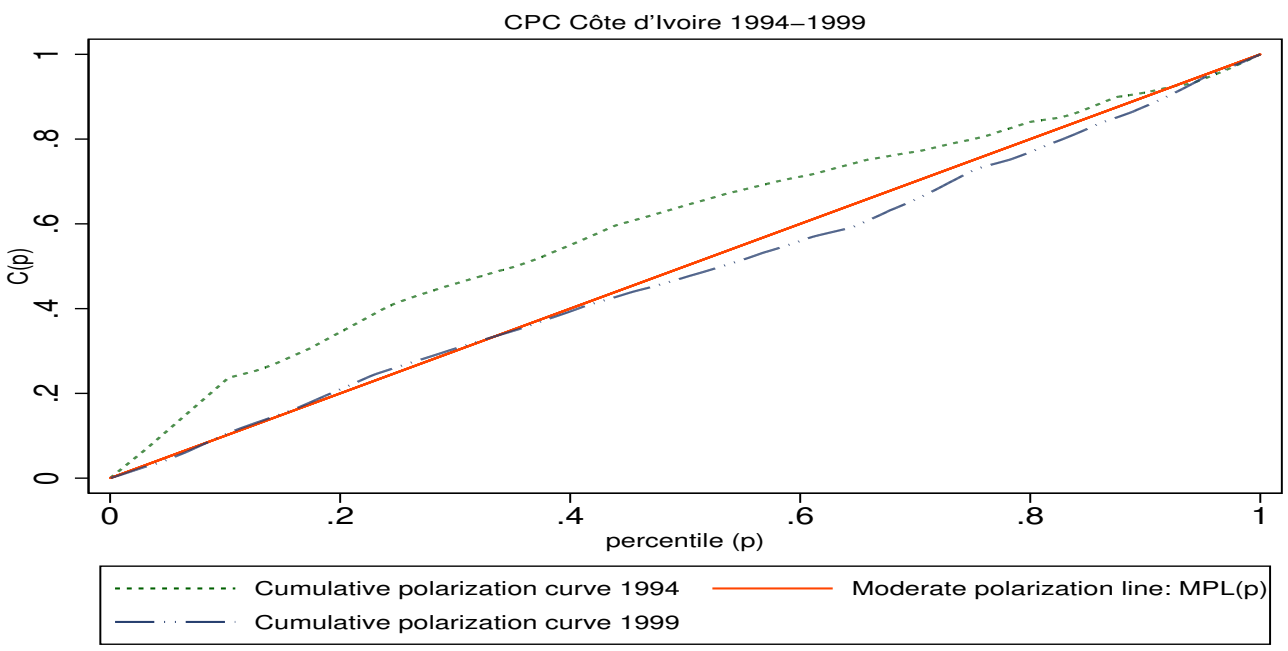

Figure 3: Côte d'Ivoire 


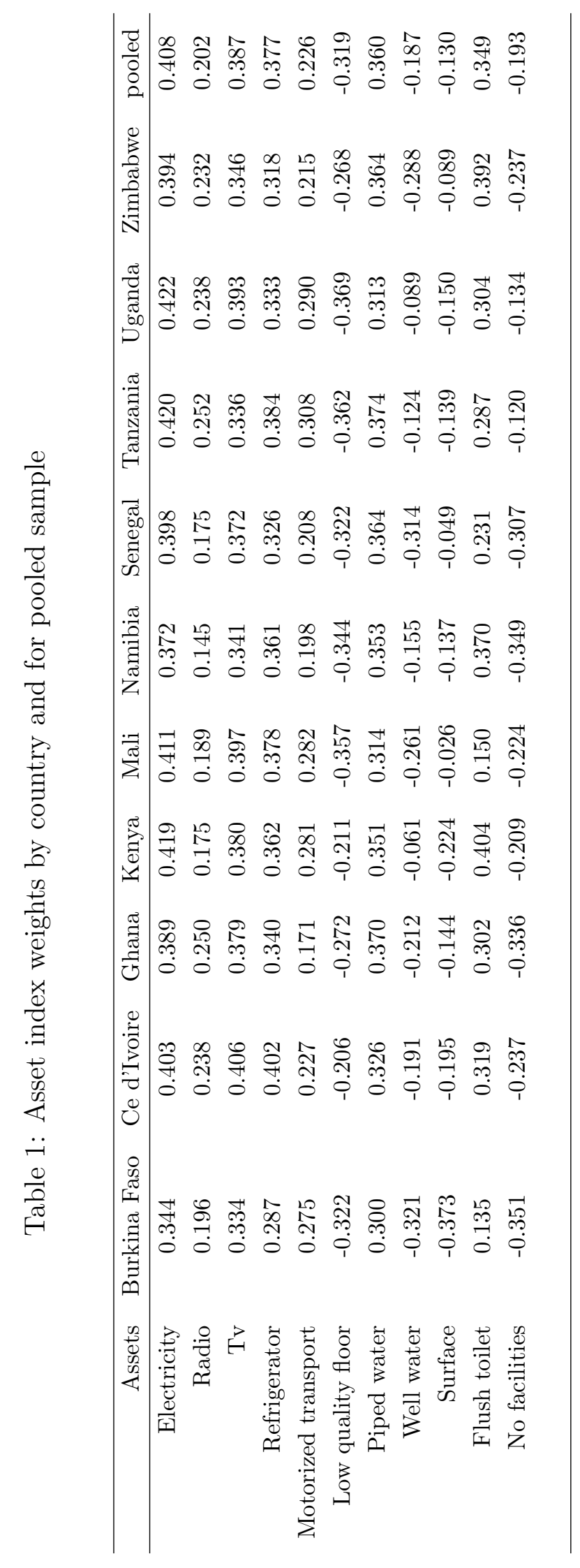


Table 2: Polarization Indices

\begin{tabular}{lcccccc}
\hline & & \multicolumn{2}{c}{ Gini: $P_{\alpha=0}$} & & \multicolumn{2}{c}{ Polarization $P_{\alpha=1}$} \\
\cline { 3 - 4 } \cline { 6 - 7 } Country & & Index & Std Dev & & Index & Std Dev \\
\hline Côte d'Ivoire & 1994 & .4193 & .0033 & & .2032 & .0038 \\
Côte d'Ivoire & 1999 & .2943 & .0022 & & .1567 & .0060 \\
Kenya & 1988 & .6098 & .0066 & & .3570 & .0033 \\
Kenya & 1998 & .5866 & .0054 & & .3090 & .0036 \\
Mali & 1995 & .4615 & .0046 & & .3277 & .0039 \\
Mali & 2001 & .4165 & .0037 & & .1961 & .0061 \\
Namibia & 1992 & .5768 & .0084 & & .3820 & .0048 \\
Namibia & 2000 & .4458 & .0058 & & .3115 & .0041 \\
Uganda & 1995 & .5431 & .0055 & & .3356 & .0039 \\
Uganda & 2000 & .4942 & .0037 & & .2544 & .0039 \\
Zimbabwe & 1994 & .5721 & .0048 & & .3009 & .0048 \\
Zimbabwe & 1999 & .5218 & .0039 & & .2717 & .0036 \\
Burkina Faso & 1992 & .5588 & .0078 & & .3568 & .0050 \\
Burkina Faso & 1998 & .6121 & .0065 & & .3661 & .0042 \\
Ghana & 1993 & .4086 & 0047 & & .2927 & .0035 \\
Ghana & 1998 & .4336 & .0041 & & .2715 & .0037 \\
Ghana & 2003 & .3791 & .0035 & & .2381 & .0031 \\
Senegal & 1992 & .4669 & .0038 & & .2255 & .0049 \\
Senegal & 1997 & .4716 & .0037 & & .2477 & .0040 \\
Tanzania & 1992 & .5296 & .0084 & & .3995 & .0050 \\
Tanzania & 1996 & .5334 & .0066 & & .3329 & .0049 \\
\hline
\end{tabular}




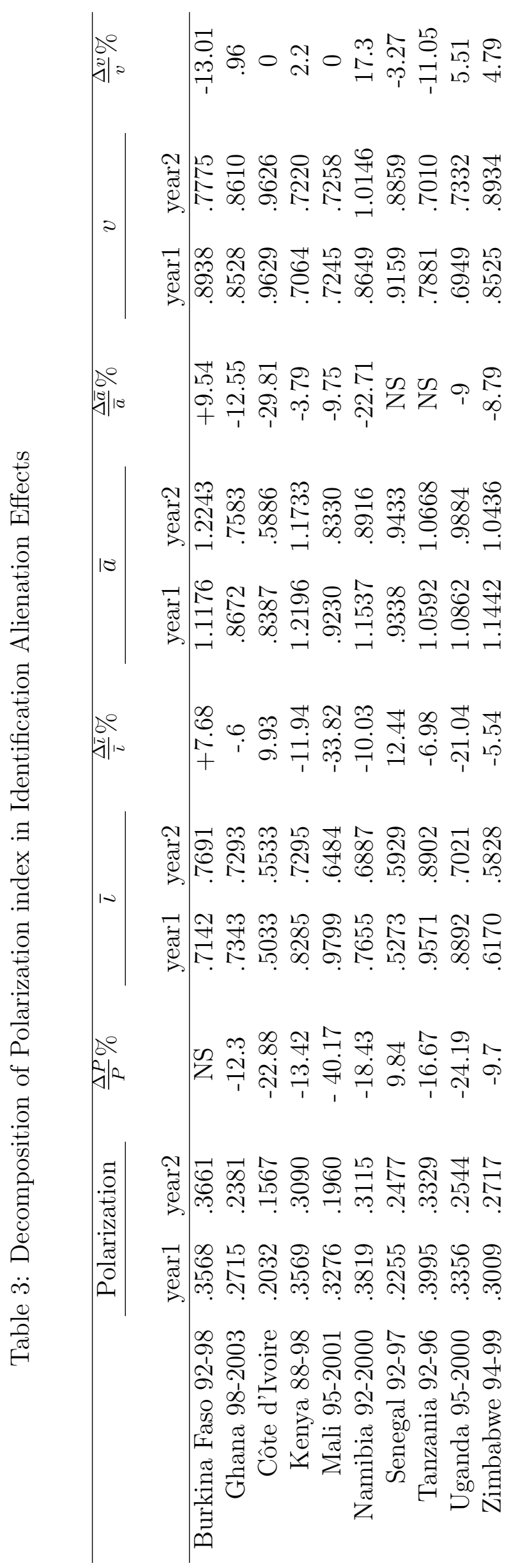


Table 4: Relative Contribution of the Two Largest Ethnic Groups and Religious Groups to the Overall Polarization

\begin{tabular}{|c|c|c|c|c|}
\hline \multirow{3}{*}{$\begin{array}{l}\text { Country } \\
\text { Burkina Faso } 1998\end{array}$} & \multicolumn{2}{|c|}{ Ethnicity } & \multicolumn{2}{|c|}{ Religion } \\
\hline & \multicolumn{2}{|c|}{ Groups / sizes in \% } & \multicolumn{2}{|c|}{ Groups / sizes in \% } \\
\hline & $\begin{array}{c}\text { Mossi } 57.5 \\
54.3 \\
\end{array}$ & $\begin{array}{c}\text { Gourmatche } 7.73 \\
11.8 \\
\end{array}$ & $\begin{array}{c}\text { Muslim } 55.35 \\
52.21 \\
\end{array}$ & $\begin{array}{l}\text { Catholic } 23.55 \\
21.16\end{array}$ \\
\hline Ghana 2003 & $\begin{array}{c}\text { Akan } 44.56 \\
40.61\end{array}$ & $\begin{array}{c}\text { Mole-Dagbani } 19.46 \\
21.9\end{array}$ & $\begin{array}{c}\text { Christian } 41.57 \\
39.93\end{array}$ & $\begin{array}{c}\text { Muslim } 17.28 \\
18.62\end{array}$ \\
\hline Côte d'Ivoire 1999 & - & - & - & - \\
\hline Kenya 1998 & $\begin{array}{c}\text { Kikuyu } 17.49 \\
14.52 \\
\end{array}$ & $\begin{array}{c}\text { Kalenjin } 16.68 \\
18.39\end{array}$ & $\begin{array}{c}\text { Protestant } 63.91 \\
64.84 \\
\end{array}$ & $\begin{array}{c}\text { Catholic } 26.95 \\
27.4\end{array}$ \\
\hline Mali 2001 & $\begin{array}{c}\text { Bambara } 30.27 \\
30.08 \\
\end{array}$ & $\begin{array}{c}\text { Sarokole/Soninke } 14.85 \\
14.35 \\
\end{array}$ & $\begin{array}{c}\text { Muslim } 93.31 \\
93 \\
\end{array}$ & $\begin{array}{c}\text { Christian } 3.49 \\
3.84 \\
\end{array}$ \\
\hline Namibia 2000 & $\begin{array}{c}\text { Oshiwambo } 34.9 \\
36.08 \\
\end{array}$ & $\begin{array}{c}\text { Damara/Nama } 21.25 \\
15.12\end{array}$ & $\begin{array}{c}\text { Protestant } 73.28 \\
74.02 \\
\end{array}$ & $\begin{array}{c}\text { Catholic } 23.4 \\
22.66 \\
\end{array}$ \\
\hline Senegal 1997 & $\begin{array}{c}\text { Wolef/Lebou } 33.79 \\
29.76\end{array}$ & $\begin{array}{c}\text { Poular } 24.35 \\
26.06 \\
\end{array}$ & - & - \\
\hline Tanzania 1996 & - & - & $\begin{array}{c}\text { Muslim } 38.91 \\
34.36 \\
\end{array}$ & $\begin{array}{c}\text { Catholic } 30.28 \\
31.42 \\
\end{array}$ \\
\hline Uganda 2000 & - & - & $\begin{array}{c}\text { Catholic } 39.48 \\
39.76\end{array}$ & $\begin{array}{c}\text { Protestant } 40.83 \\
41.78\end{array}$ \\
\hline Zimbabwe 1999 & - & $\begin{array}{l}- \\
-\end{array}$ & $\begin{array}{c}\text { Christian } 81.12 \\
80.38\end{array}$ & $\begin{array}{c}\text { None } 11.58 \\
11.49\end{array}$ \\
\hline
\end{tabular}

Note: Relative contribution to overall polarization appears in every second line 
Table 5: Polarization and Gini Indices: Pooled Sample

\begin{tabular}{lccccc}
\hline & \multicolumn{2}{c}{ Gini } & & \multicolumn{2}{c}{ Polarization } \\
\cline { 2 - 3 } \cline { 5 - 6 } Country & & & & \\
\hline Burkina Faso 1998 & .6216 & 1 & & .2958 & 4 \\
Ghana 1998 & .4656 & 8 & & .2127 & 8 \\
Côte d'Ivoire 1999 & .3599 & 10 & & .1664 & 10 \\
Kenya 1998 & .5012 & 4 & & .2981 & 3 \\
Mali 2001 & .4916 & 5 & & .2109 & 9 \\
Namibia 2000 & .4761 & 7 & & .3096 & 2 \\
Senegal 1997 & .5366 & 3 & & .2400 & 7 \\
Tanzania 1996 & .4835 & 6 & & .3282 & 1 \\
Uganda 2000 & .4577 & 9 & & .2939 & 5 \\
Zimbabwe 1999 & .5446 & 2 & .2647 & 6 \\
\hline
\end{tabular}

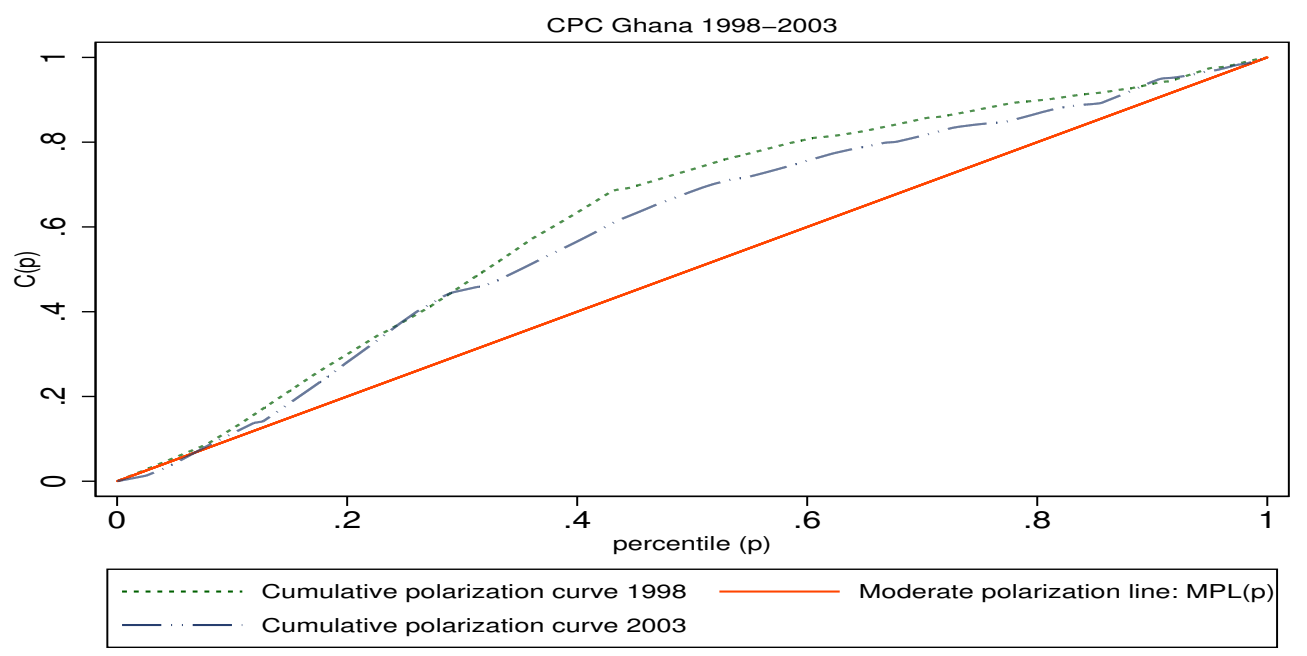

Figure 4: Ghana 


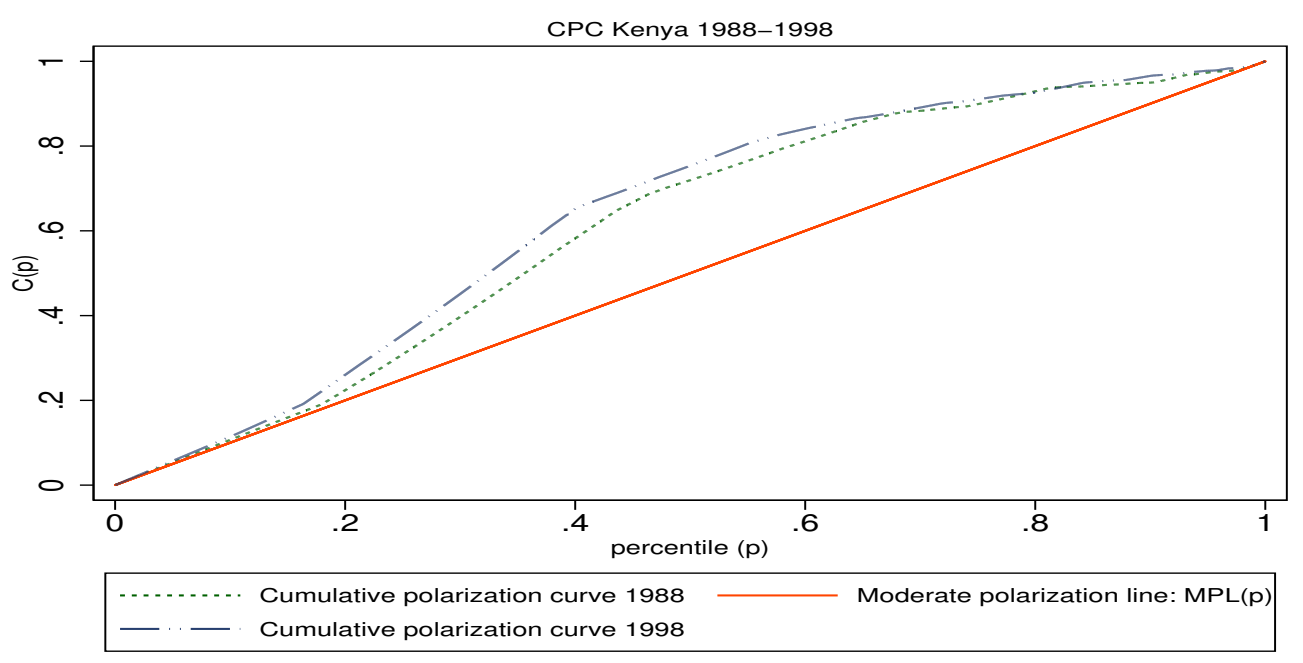

Figure 5: Kenya

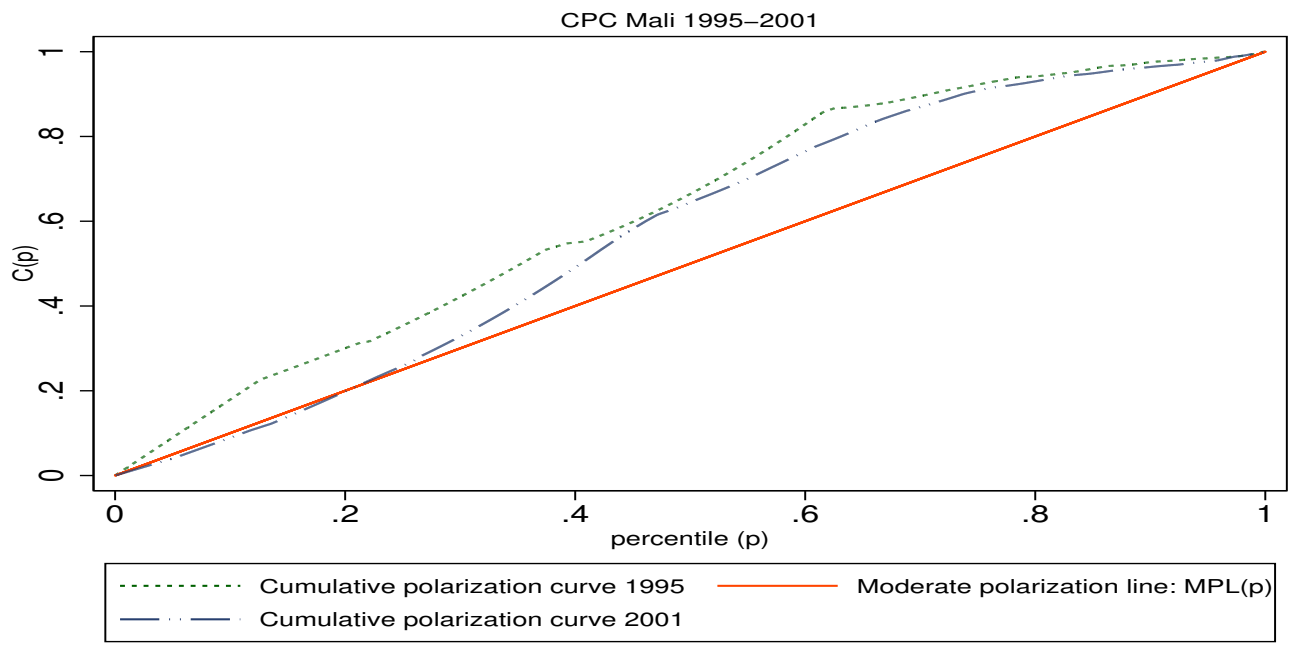

Figure 6: Mali 


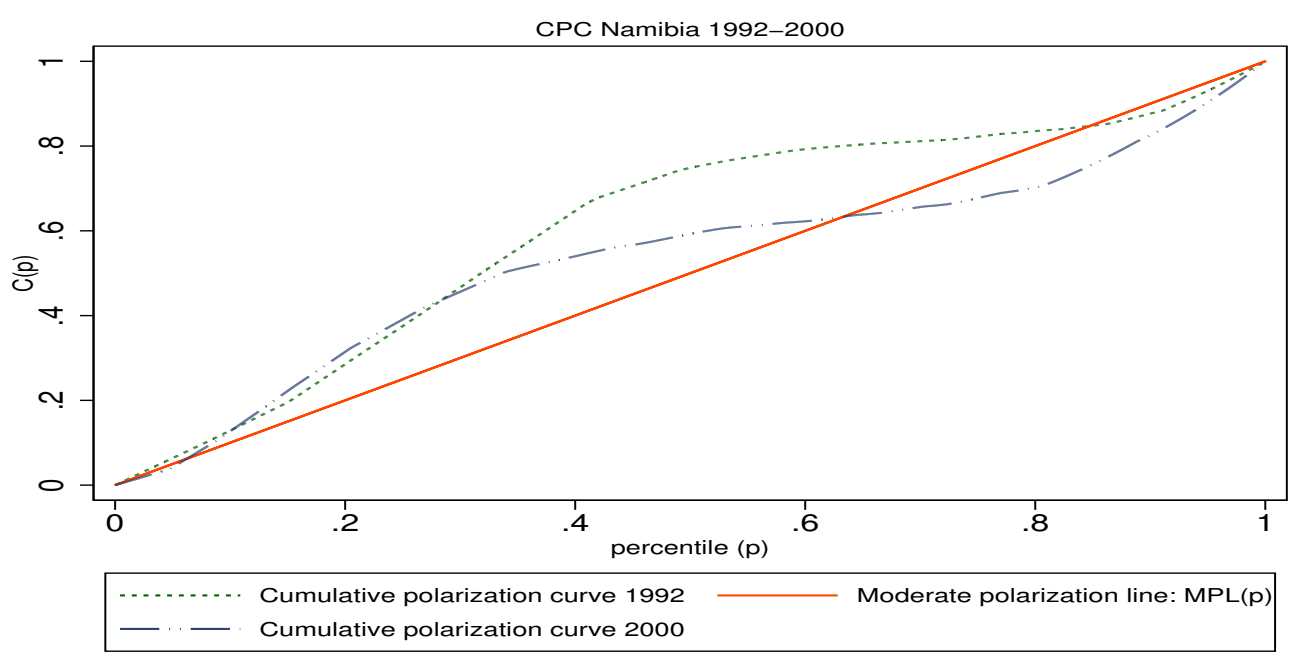

Figure 7: Namibia

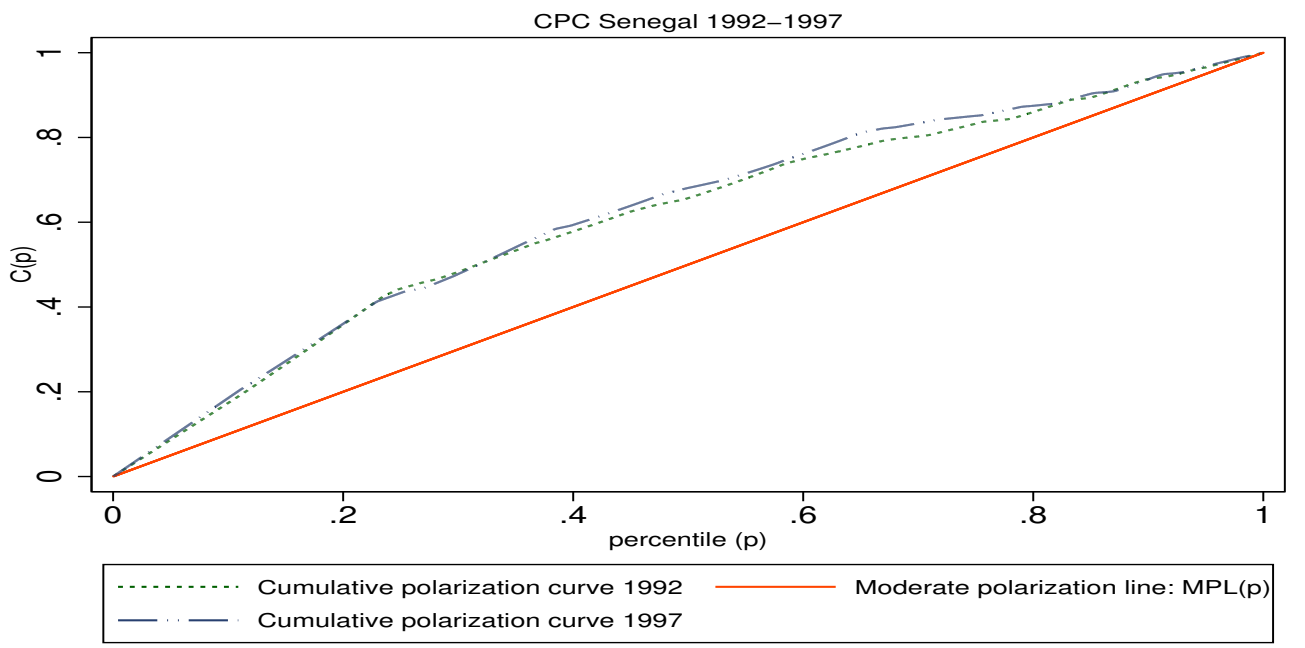

Figure 8: Senegal 


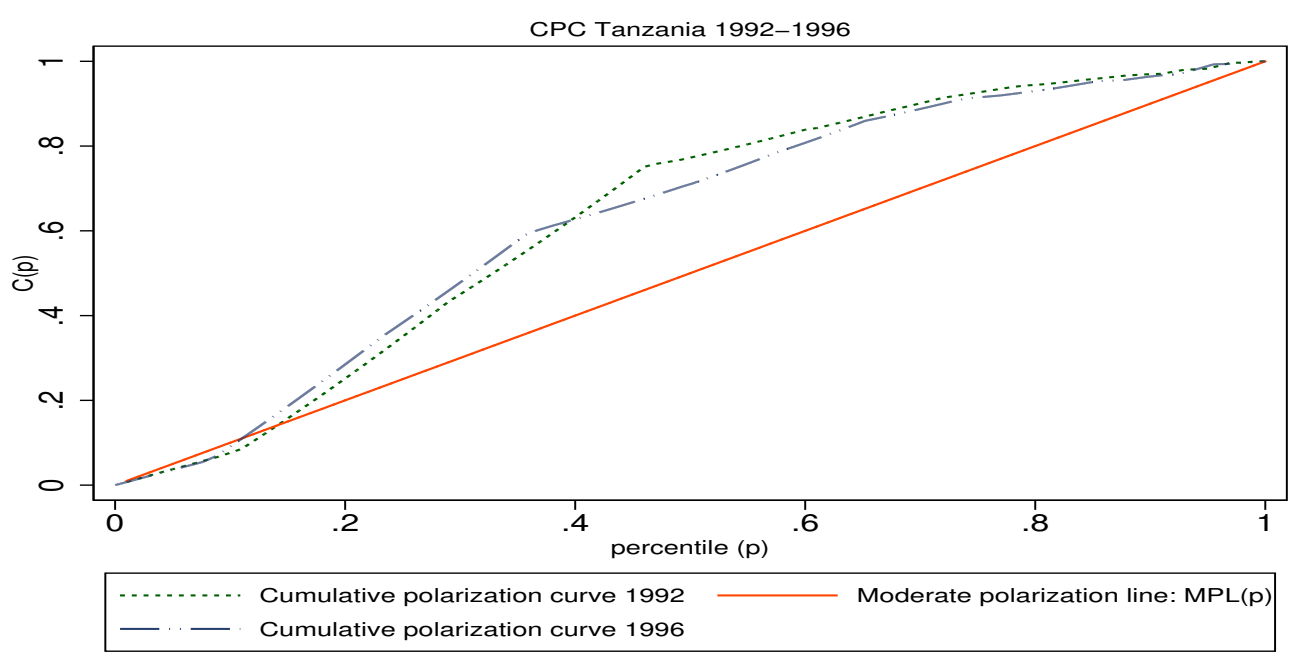

Figure 9: Tanzania

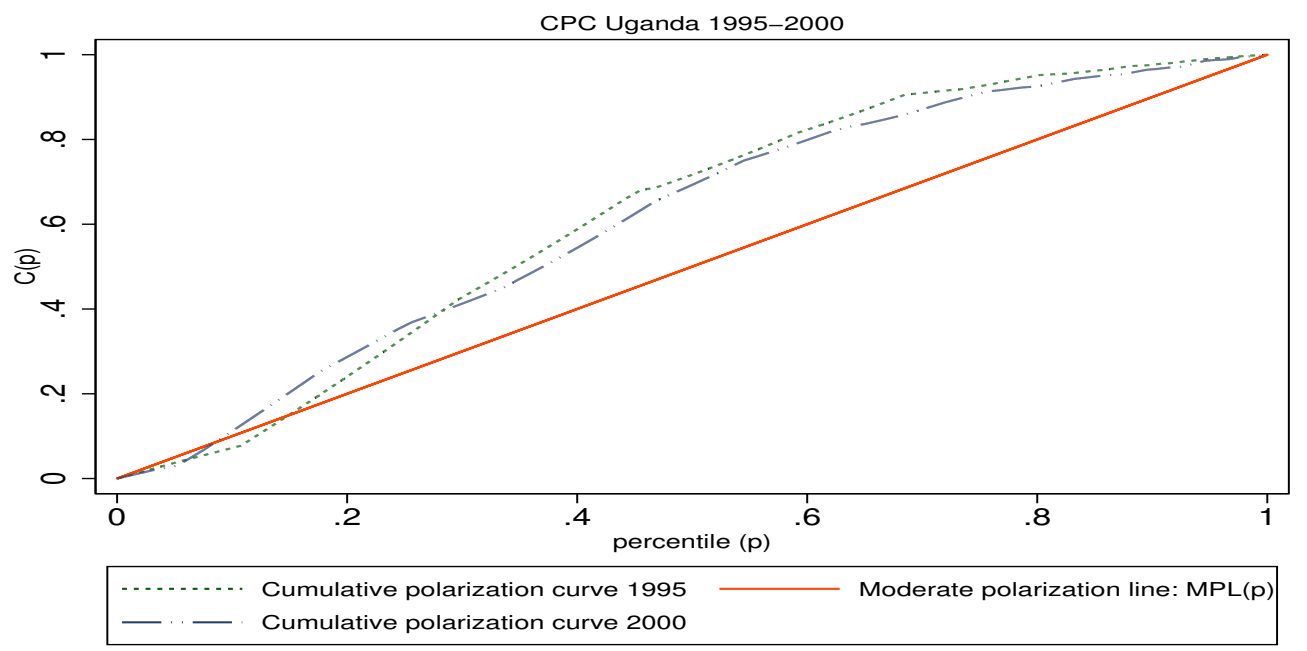

Figure 10: Uganda 


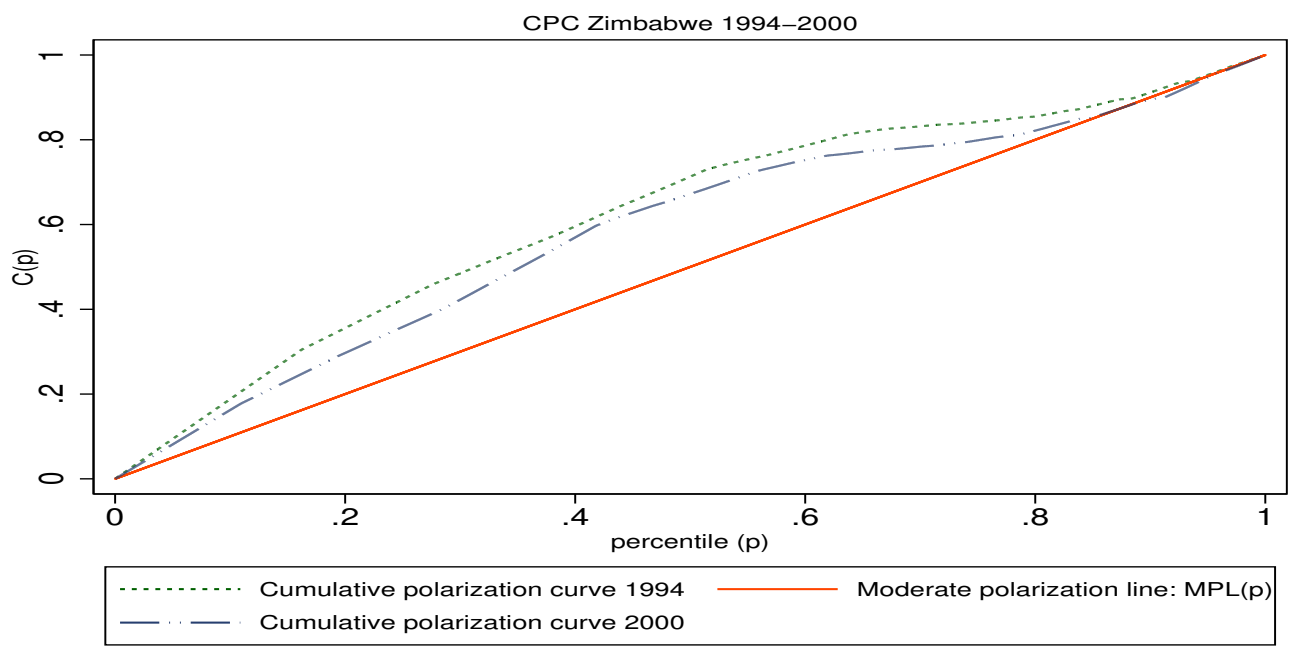

Figure 11: Zimbabwe 